${ }^{1}$ Национальный медицинский исследовательский центр нейрохирургии имени академика Н.Н. Бурденко, Москва, Россия

${ }^{2}$ Национальный медицинский исследовательский центр эндокринологии, Москва, Россия

В настоящее время терапия каберголином является основным методом лечения пролактином. Использование препарата в большинстве случаев приводит к регрессу опухоли, нормализации уровня пролактина и восстановлению гонадотропной функции. Механизм его действия на опухолевые клетки in vivo, в динамике прослеженный в одной и той же опухоли человека, представляет значительный интерес.

Мы наблюдали пациента 30 лет, который был оперирован двукратно по поводу гигантской пролактиномы до и на фоне приема каберголина. При морфологическом исследовании после первой операции (до терапии каберголином) выявлена пролактинпозитивная опухоль гипофиза с индексом мечения Кі-67 - 8\% и с выраженной экспрессией рецепторов дофамина 2 типа (D2R), CD31 и CD34. Через 4 месяца, в течение которых пациент получал каберголин в дозе начиная с 0,5 мг до 1,5 мг в неделю, проведена повторная операция транссфеноидальным доступом с субтотальным удалением остаточной ткани опухоли. При морфологическом исследовании второго биоптата опухоль сохраняла выраженную иммунопозитивность к пролактину и D2R, при этом отмечено снижение индекса мечения Кі-67 до 2\%, а также снижение экспрессии CD31 и CD34. Последующая терапия каберголином привела к стойкой нормопролактинемии, восстановлению андрогенного (и репродуктивного) статуса и отсутствию рецидива опухоли в течение 10-летнего периода на фоне лечения каберголином. Таким образом, одним из механизмов действия каберголина, приводящим к регрессу опухоли, является снижение пролиферативного индекса и ангиогенеза опухоли.

КЛЮЧЕВЫЕ СЛОВА: гигантская пролактинома; каберголин; пролиферативный потенциал; Кі-67; агрессивная аденома гипофиза; пролактинома у мужчин; классификачия ВОЗ опухолей гипофиза.

\title{
CHANGES IN THE MORPHOLOGICAL STRUCTURE OF GIANT PROLACTINOMA DURING TREATMENT WITH CABERGOLINE
}

(c) Lyudmila I. Astaf'eva1*, Lyudmila V. Shishkina', Pavel L. Kalinin'1, Boris A. Kadashev'1, Julia G. Sidneva', Oleg I. Sharipov'1, Galina A. Melnichenko²

${ }^{1}$ N.N. Burdenko National Medical Research Center of Neurosurgery, Moscow, Russia

${ }^{2}$ Endocrinology Research Centre, Moscow, Russia

Currently, cabergoline therapy is the main method of treatment with prolactin. The use of the drug in most cases leads to tumor regression, normalization of prolactin levels and restoration of gonadotropic function. The mechanism of its impact on tumor cells in vivo, which is dynamically traced in the same human tumor, is the case of considerable interest. We observed a 30-year-old patient who was operated on twice for a giant prolactinoma before and on treatment by cabergoline. The morphological study after the first surgery (before introducing of cabergoline therapy) revealed a prolactin-positive pituitary tumor with a Ki-67 labeling index of $8 \%$ and with strong expression of dopamine type 2 receptors (D2R), CD31 and CD34. After 4 months, during which the patient received cabergoline at a dose starting from $0.5 \mathrm{mg}$ to $1.5 \mathrm{mg}$ per week, a second transsphenoidal surgery was performed with subtotal removal of residual tumor tissue. During the morphological study of the second biopsy sample, the tumor retained a pronounced immunopositivity to prolactin and D2R, with a decrease in the labeling index $\mathrm{Ki}-67$ to $2 \%$, as well as a decrease in the expression of CD31 and CD34. Subsequent cabergoline therapyresulted in persistent normoprolactinemia, restoration of androgen (and reproductive) status, and no tumor recurrence over a 10-year period on cabergoline treatment. Thus, one of the mechanisms of effect of cabergoline that leads to tumor regression is a decrease in the proliferative index and angiogenesis of the tumor.

KEYWORDS: giant prolactinoma; cabergoline; proliferative potential; Ki-67; aggressive pituitary adenoma; prolactinoma in men; classification of pituitary tumors by WHO. 


\section{АКТУАЛЬНОСТЬ}

Пролактиномы составляют около 40\% всех опухолей гипофиза. Среди них преобладают микроаденомы гипофиза, которые выявляются преимущественно у женщин. Макропролактиномы встречаются гораздо реже и менее изучены. Эта группа аденом неоднородна по своему составу и различается по размеру опухолей, темпам их роста, клинической симптоматике. Если небольшие опухоли эндоселлярной локализации могут сопровождаться лишь клинической картиной гиперпролактинемии и чаще выявляются у женщин, то пролактиномы гигантских размеров склонны к быстрому и инфильтративному росту, часто имеют многоузловой характер строения, проникают в желудочки мозга, кавернозные синусы, основную пазуху, носоглотку, вызывают неврологические и зрительные нарушения; описаны в основном у мужчин и для лечения представляют самую сложную группу из всех пролактином $[1,2,3]$. До момента появления агонистов дофамина хирургическое лечение было основным методом лечения. Успехи современной фармакологии позволили вплотную подойти к альтернативному решению этой проблемы. В настоящее время терапия каберголином является основным методом лечения пролактином, приводящим к уменьшению размеров опухоли, нормализации уровня пролактина (ПРЛ) у большинства пациентов $[4,5]$. Впервые в литературе уменьшение опухоли гипофиза в ходе лечения агонистами дофамина описано в начале 1970-х гг. [6]. В последующих многочисленных исследованиях in vitro на лабораторных животных, а также в опухолях человека было показано, что препарат избирательно активирует рецепторы дофамина 2 типа, что сопровождается уменьшением размера опухоли, а также синтеза и секреции ПРЛ. Было показано, что регресс опухоли может быть обусловлен различными механизмами, в том числе уменьшением размера клеток $[7,8,9]$, индукцией апоптоза и некрозом опухоли $[10,11]$, а также подавлением клеточной пролиферации [12]. Однако изучение эффектов каберголина in vivo непосредственно на клеточные структуры одной и той же опухоли гипофиза человека представляет значительный интерес.

\section{ОПИСАНИЕ СЛУЧАЯ}

Мужчина 30 лет обратился в НМИЦН им. ак. Н.Н. Бурденко в ноябре 2004 г. с жалобами на ухудшение зрения, головные боли. При проведении МРТ выявлена аденома гипофиза гигантских размеров.

Из анамнеза: в течение 3 лет отмечает повышение массы тела на 20 кг, повышение АД до 150/100 мм рт. ст., снижение полового влечения, нарушение эректильной функции; в течение месяца появление жалоб на ухудшение зрения.

Амбулаторное обследование, проведенное в НМИЦН им. ак. Н.Н. Бурденко, позволило диагностировать опухоль эндо-супра-латеро-инфраселлярной локализации гигантских размеров, максимальный размер которой составил 6,7 см (рис. 1 a, b); зрительные нарушения в виде битемпоральной гемианопсии и снижение остроты зрения правого глаза (VIS OD=0,4), VIS OS=1,0, небольшой правосторонний экзофтальм в 1,5 мм (выстояние $\mathrm{OD}=18$ мм, OS=16,5 мм). Уровень ПРЛ был более 5000 мЕд/л (30-360) (исследование с разведением сыворотки для определения ПРЛ не проводилось), тестостерона - 2,3 (8-35) нмоль/л, тиреотропного гормона - 1,3 (0,4-4,0) мЕд/л, св. Т4 - 12 (9-22) нмоль/л, кортизола - 398 (260-720) нмоль/л.

Учитывая гигантские размеры опухоли, выраженное снижение зрительных функций, а также приоритетный В то время принцип нейрохирургического лечения крупных опухолей вне зависимости от гормональной активности (до активного внедрения в практику каберголина), предпринято проведение операции двухэтапным методом (транскраниальным и трансназальным доступами с интервалом в 3 месяца между операциями).

В ноябре 2004 г. проведена операция транскраниальным доступом с удалением супраселлярной части опухоли. После операции отмечено ухудшение зрения правого глаза до амавроза, одновременно появился правосторонний птоз и ограничение объема движений правого глазного яблока (грубый парез III нерва справа). Уровень ПРЛ после операции оставался более 5000 мЕд/л (30-360).

При морфологическом исследовании удаленная опухоль была представлена разрастаниями преимущественно округлых клеток со светлой цитоплазмой, гиперхромными ядрами и единичными митозами. В ткани опухоли отмечалась повышенная васкуляризация, опухолевые клетки располагались между многочисленных сосудов с расширенным просветом и утолщенными фиброзированными стенками (рис. 2а) При иммуногистохимическом исследовании были выявлены четко выраженная положительная экспрессия ПРЛ, а также отчетливая экспрессия рецепторов дофамина 2 типа (D2R), повышенная пролиферативная активность опухолевых клеток, индекса мечения (ИМ) Кі-67 до 8\% (рис. 2c), высокая экспрессия CD34 (рис. 2е). Опухоль была иммунонегативна к ТТГ, соматотропному, лютеинизирующему, фолликулостимулирующему, адренокортикотропному гормонам.

После операции пациенту назначена терапия каберголином в дозе 0,5 мг с постепенным повышением дозы до 1,5 мг в неделю, на этом фоне было отмечено снижение уровня ПРЛ до 3800 мЕд/л (30-360).

При контрольном обследовании через 3 месяца регрессировал парез правого глазодвигательного нерва, однако сохранялась слепота на правый глаз. При МРТисследовании (рис. 1 c, d) определялся остаток опухоли эндо-инфраселлярной локализации.

В феврале 2005 г. проведено субтотальное удаление эндо-инфраселлярного остатка опухоли трансназальным транссфеноидальным доступом. При нейроофтальмологическом осмотре после операции зрительные нарушения оставались без динамики. Уровень ПРЛ в крови после операции снизился до 1304 мЕд/л (30-360).

При морфологическом исследовании обращало на себя внимание изменение структуры опухоли, выражавшееся в увеличении солидного компонента с периваскулярными структурами и уменьшении количества сосудов, которые были представлены преимущественно 
сосудами синусоидного типа с узким просветом. Фигур митотического деления не выявлено (рис. 2b). При этом опухоль сохраняла такую же выраженность иммунопозитивности к ПРЛ и D2R, как и в первом биоптате. Пролиферативный потенциал опухоли был значительно ниже, ИМ Кі-67 около 2\% (рис. 2d), а также отмечено уменьшение количества сосудов, оцененных с помощью экспрессии CD34 (рис. 2f).
После операции продолжена терапия каберголином в дозе 1,5 мг с последующим ее снижением до 0,25 мг в неделю, которая привела к стойкой нормопролактинемии, восстановлению андрогенного статуса и отсутствию рецидива опухоли в течение последующего 10-летнего периода наблюдения (рис. 1 e, f). Через 3 года после операции у жены пациента наступила беременность, закончившаяся рождением здорового ребенка.
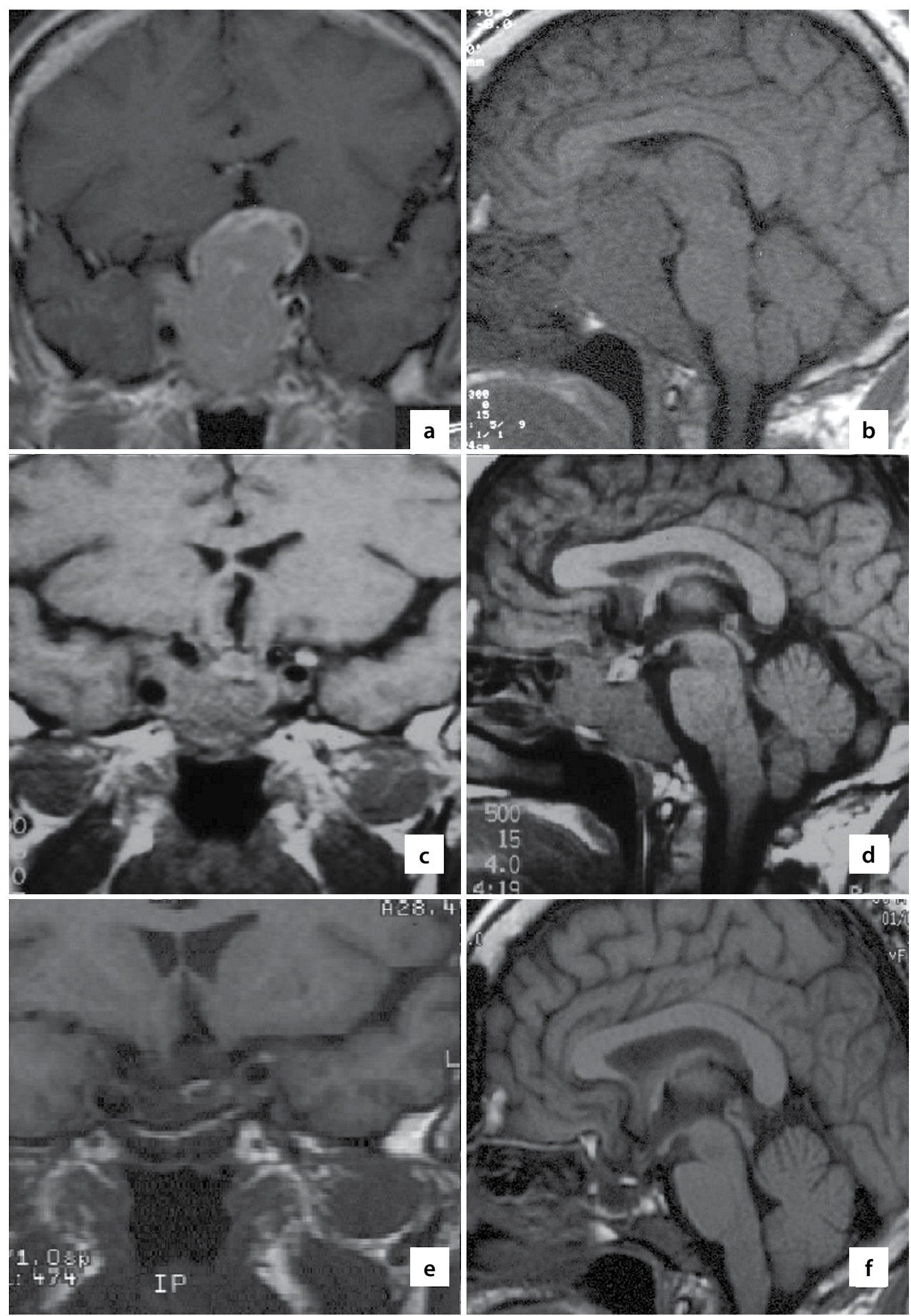

Рисунок 1. МРТ во фронтальной и сагиттальной проекциях.

a, b. Перед 1-м этапом хирургического лечения (удаление транскраниальным доступом). Визуализируется гигантская аденома гипофиза эндо-супра-латеро-инфраселлярной локализации.

c, d. Через 3 мес после удаления опухоли транскраниальным доступом и курса терапии каберголином перед 2-м этапом хирургического лечения (удаление транссфеноидальным доступом). Визуализируется остаток опухоли эндо-латеро-инфраселлярной локализации.

е, f. Через 10 лет после удаления опухоли на фоне терапии каберголином. Регресс опухоли и «пустое» турецкое седло. 


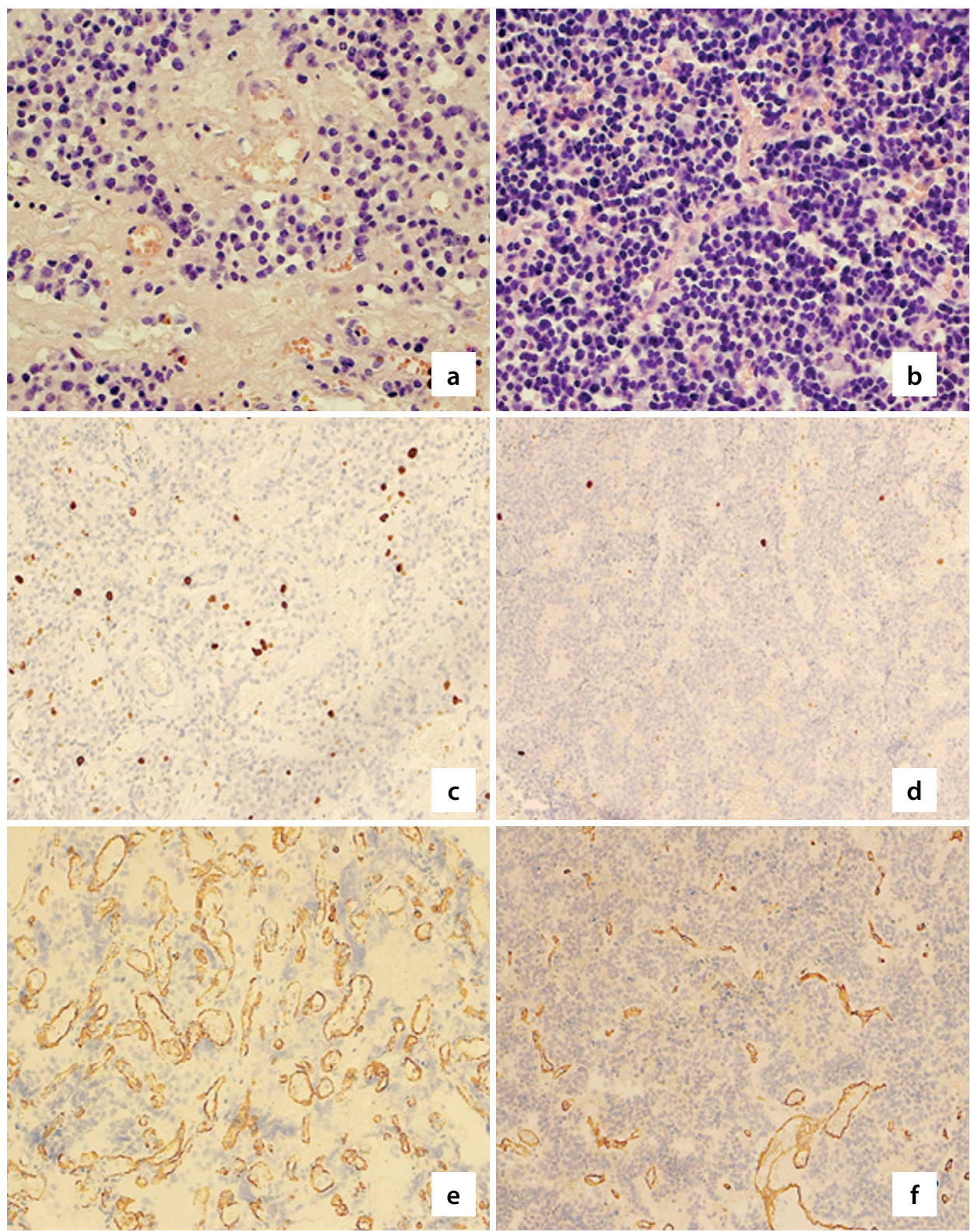

Рисунок 2. Морфологические и иммуногистохимические характеристики гигантской пролактиномы до и после терапии каберголином у пациента 30 лет.

а. Аденома гипофиза (после первой операции, до терапии каберголином): опухолевые клетки располагаются между сосудами с утолщенными стенками. Окраска гематоксилином и эозином; $\times 400$

b. Аденома гипофиза солидного строения с тонкостенными сосудами синусоидного типа (после второй операции, на фоне терапии каберголином). Окраска гематоксилином и эозином; $\times 200$.

c. Иммуноэкспрессия Ki-67 в ядрах клеток опухоли (после первой операции, до терапии каберголином): индекс пролиферации Кі-67 8\%, $\times 200$.

d. Иммуноэкспрессия Кі-67 в ядрах клеток опухоли (после второй операции, на фоне терапии каберголином): пролиферация Кі-67 $2 \%$, $\times 200$.

е. Иммуноэкспрессия CD34 в эндотелии сосудов опухоли демонстрирует богатую сосудистую сеть, ×200 (после первой операции, до терапии каберголином).

f. Иммуноэкспрессия CD34 в сосудистом эндотелии опухоли показывает более редкие мелкие сосуды с тонкими стенками по сравнению с первичной опухолью, ×100 (после второй операции, на фоне терапии каберголином).

\section{ОБСУЖДЕНИЕ}

До появления каберголина хирургическое лечение было основным методом лечения гигантских аденом гипофиза вне зависимости от гормональной активности. При этом одной из методик удаления опухолей с выраженным супраселлярным ростом и массивной инвазией в структуры основания черепа были двухэтапные операции, когда раздельно применялись транскраниальный и транссфеноидальный доступы к разным отделам опухоли, внедряющейся как в полость, так и в структуры основания черепа [13, 14].
С момента активного внедрения (с начала 2000-х гг.) в практику высокоэффективного и безопасного препарата каберголина из группы агонистов дофамина с пролонгированным действием, основным методом лечения пролактином, в том числе гигантских размеров, стало медикаментозное лечение.

В многочисленных исследованиях in vitro, а также на лабораторных животных было показано, что препарат избирательно активирует D2R на поверхности клетки, что приводит к подавлению транскрипции и экспрессии гена ПРЛ, а также к уменьшению синтеза и секреции ПРЛ [15-20]. 
В различных исследованиях было показано, что регресс опухоли обусловлен подавлением клеточной пролиферации клеток [12]. Одним из наиболее достоверных и признанных в мире маркеров клеточной пролиферации является ИМ Кi-67. Избыточная экспрессия ИМ Ki-67 отражает агрессивные характеристики и является индикатором рецидивирования опухоли [21, 22].

Исследования, оценивающие показатели ИМ Кі-67 в аденомах человека, леченных агонистами дофамина, немногочисленны. В работах Ekramullah S.M., Stefaneanu L. проводились исследования двух групп пациентов с пролактиномой: оперированных без предшествующего лечения и с дооперационным лечением агонистами дофамина. Показано, что терапия агонистами дофамина приводила к достоверному снижению уровня Кі-67 [19, 23]. Однако в этих исследованиях пролиферативный потенциал оценивался в разных группах пациентов. Последующие исследования выполнялись преимущественно у пациентов с фармакорезистентными опухолями $[24,25]$. Данных о влиянии каберголина на пролиферативный потенциал опухоли при хорошей чувствительности к терапии in vivo практически нет.

Действительно, в настоящее время первым и основным методом лечения пролактином является терапия каберголином. На фоне положительного ответа аденомы на лечение в виде уменьшения ее размеров и снижения уровня ПРЛ это лечение продолжают вплоть до полного регресса опухоли и нормализации уровня ПРЛ. Такие пациенты не нуждаются в хирургическом лечении, и, следовательно, получение гистологического материала не представляется возможным. Хирургическому лечению в основном подвергаются фармакорезистентные опухоли, а также аденомы, лечение которых сопровождается развитием осложнений, преимущественно назальной ликвореи [26, 27].

Показано отсутствие влияния хирургического вмешательства на опухоль - по данным литературы, Кі-67 сохранялся в прежних значениях после удаления рецидива аденомы гипофиза [28, 29].
Поэтому практически невозможно оценить влияние каберголина на пролиферативный индекс одной и той же чувствительной к терапии опухоли in vivo.

Мы описали пациента, который был оперирован на стыке первичного хирургического и медикаментозного подходов в лечении гигантских пролактином. Поэтомy in vivo мы смогли оценить влияние каберголина на пролиферативный потенциал одной и той же опухоли. В биоптатах, взятых при первой и второй операциях, опухоль сохраняла выраженную иммунопозитивность к ПРЛ и D2R. Однако отмечено значимое снижение ИМ Ki-67 с 8\% до 2\% и снижение экспрессии CD31и CD34, а также изменение структуры сосудов опухоли (табл. 1).

Ангиогенез - процесс образования новых кровеносных сосудов - является важным звеном в патогенезе развития различных опухолей, в том числе аденом гипофиза. Опухоли гипофиза менее васкуляризированы в сравнении с неопухолевой гипофизарной тканью; предполагается, что отсутствие значительного ангиогенеза лежит в основе медленного роста опухолей гипофиза, а прогрессирование опухолевых клеток должно коррелировать с ангиогенезом. Параметром для оценки ангиогенеза является плотность сосудов в опухоли. Было показано, что плотность сосудов значительно выше в макро- в сравнении с микропролактиномами; в инвазивных пролактиномах в сравнении с неинвазивными; а также в ПРЛ-секретирующих карциномах гипофиза. В ранее проведенных исследованиях было показано, что опухоли, леченные агонистами дофамина, имеют более низкую плотность сосудов в сравнении с первично-оперированными пролактиномами без предшествовавшего лечения каберголином [3, 19].

С целью оценки ангиогенеза аденомы до и на фоне лечения каберголином мы провели исследование с помощью антител к маркерам сосудистого эндотелия CD34 и CD31. Выявлено, что до лечения каберголином плотность сосудов в аденоме, оцененная по степени экспрессии CD34 и CD31, была значимо выше, чем в биопсии при второй операции. Кроме этого, изменилась сама структура сосудов опухоли.

Таблица 1. Морфологические и иммуногистохимические характеристики гигантской пролактиномы до и после терапии каберголином у пациента 30 лет.

\begin{tabular}{lll}
\hline $\begin{array}{c}\text { Морфологическое } \\
\text { исследование опухоли }\end{array}$ & \multicolumn{1}{c}{$\begin{array}{c}\text { При 1-й операции } \\
\text { (до лечения каберголином) }\end{array}$} & \multicolumn{1}{c}{$\begin{array}{c}\text { При 2-й операции } \\
\text { (после лечения каберголином) }\end{array}$} \\
\hline \multirow{2}{*}{ Световая микроскопия } & Клеточные комплексы разделены & Опухоль солидного строения \\
& $\begin{array}{l}\text { многочисленными сосудами с расширенным } \\
\text { просветом и утолщенными стенками }\end{array}$ & $\begin{array}{l}\text { с тонкостенными сосудами } \\
\text { синусоидного типа }\end{array}$ \\
Митозы & Наличие & Отсутствие \\
Васкуляризация опухоли & Высокая & Низкая \\
\hline
\end{tabular}

Иммуногистохимические характеристики опухоли

\begin{tabular}{lcc}
\hline Экспрессия ПРЛ & ++ & ++ \\
Экспрессия D2R & ++ & ++ \\
Экспрессия ИМ Кi-7 & $8 \%$ & ++ \\
Экспрессия СD31 & + \\
Экспрессия СD34 & ++ \\
\hline
\end{tabular}

+ - слабовыраженная экспрессия;

++ - выраженная экспрессия. 
B 1982 г. Landolt A.M. и соавт. описали развитие периваскулярного фиброза у пациентов на фоне длительного лечения бромокриптином [30]. Этим феноменом некоторые авторы объясняли худшие результаты хирургического лечения пациентов с пролактиномой, получавших бромокриптин [31]. В нашем клиническом наблюдении после 3-месячного курса терапии каберголином мы не выявили подобных изменений в опухоли. Напротив, если до лечения сосуды имели расширенный просвет и утолщенные фиброзированные стенки (рис. 2 а), то в биоптате, полученном при второй операции, они были преимущественно представлены сосудами синусоидного типа с тонкими стенками и узким просветом (рис. 2 b).

Согласно предыдущей версии классификации ВО3 опухолей эндокринной системы, применяемой с 2004 по 2017 гг., выделяли «типичные» и «атипичные» аденомы гипофиза. К последним относили опухоли с клиническими признаками инфильтративного роста и морфологическими признаками атипии в виде повышенного митотического индекса, гиперэкспрессии р53 и повышения ИМ Кі-67 свыше 3\% [32]. При использовании этих критериев показатели заболеваемости атипичными аденомами, по различным данным, варьируют от 2 до 15\%, а прогностическая ценность этой классификации еще не установлена, несмотря на длительный срок ее существования [33, 34]. Тем не менее, согласно этой классификации, в нашем наблюдении первоначально опухоль можно было трактовать как «атипичную», тогда как на фоне лечения каберголином морфологическая картина стала соответствовать «типичной» аденоме гипофизе.
В современной морфологической классификации ВОЗ опухолей эндокринной системы, принятой в 2017 г., отсутствует понятие «атипичной» аденомы гипофиза, вместо него предложен термин «аденома с повышенной пролиферативной активностью». Кроме того, в новой классификации также выделены подтипы опухолей гипофиза, обладающих клинически агрессивным течением, в том числе «пролактиномы у мужчин» $[35,36]$.

\section{ЗАКЛЮЧЕНИЕ}

В описанном нами наблюдении терапия каберголином привела к значительному клиническому улучшению, подтвержденному морфологическими изменениями в опухолевой ткани: снижению пролиферативного потенциала клеток, что является одним из механизмов уменьшения размеров опухоли, а также снижению сосудистой плотности и структуры сосудов опухоли.

\section{ДОПОЛНИТЕЛЬНАЯ ИНФОРМАЦИЯ}

Источник финансирования. Статья подготовлена на личные средства авторского коллектива.

Согласие пациента. Пациент добровольно подписал информированное согласие на публикацию в журнале «Проблемы эндокринологии» персональной медицинской информации в обезличенной форме.

Конфликт интересов. Авторы декларируют отсутствие явных и потенциальных конфликтов интересов, связанных с публикацией настоящей статьи.

Участие авторов. Все авторы принимали участие в наблюдении пациента, внесли существенный вклад в подготовку статьи, прочли и одобрили финальную версию перед публикацией.

\section{СПИСОК ЛИТЕРАТУРЫ | REFERENCES}

1. Corsello SM, Ubertini G, Altomare M, et al. Giant prolactinomas in men: efficacy of cabergoline treatment. Clin Endocrinol (Oxf). 2003;58(5):662-670. doi: 10.1046/j.1365-2265.2003.01770.x.

2. Shrivastava RK, Arginteanu MS, King WA, et al. Giant prolactinomas: clinical management and long-term follow up. J Neurosurg. 2002;97(2):299-306. doi: 10.3171/jns.2002.97.2.0299.

3. Ines M, Millán P, Cristina C, et al. Role of VEGF, FGF-2 and CD31(2014). In: Hayat M.A., ed. Tumors of the central nervous system. Vol. 12. Molecular mechanisms, children's cancer, treatments, and radiosurgery. Springer, Dordrecht; 2014. P. 33-41. doi: 10.1007/978-94-007-7217-5_3.

4. Shimon I. Giant prolactinomas: Multi-modal approach to achieve tumor control. Endocrine. 2017;56(2):227-228. doi: 10.1007/s12020-016-1225-x.

5. Астафьева Л.И., Кадашев Б.А., Калинин П.Л., и др. Выбор тактики лечения гигантских пролактинсекретирующих аденом гипофиза // Вопросы нейрохирургии им. Н.Н. Бурденко. - 2009. №2. - C. 23-28. [Astafieva LI, Kadashev BA, Kalinin PL, et al. Selection of management tactics in treatment of giant prolactinsecreting pituitary adenomas. Burdenko's journal of neurosurgery. 2009;(2):23-28. (In Russ).]

6. Quadri SK, Meites J. Regression of spontaneous mammary tumors in rats by ergot drugs. Proc Soc Exp Biol Med. 1971;138(3):999-1001. doi: 10.3181/00379727-138-36036.

7. Barrow DL, Tindall GT, Kovacs K, et al. Clinical and pathological effects of bromocriptine on prolactin-secreting and other pituitary tumors. J Neurosurg. 1984;60(1):1-7. doi: 10.3171/jns.1984.60.1.0001

8. Rengachary SS, Tomita T, Jefferies B, et al. Structural changes in human pituitary tumor after bromocriptine therapy. Neurosurgery. 1982;10(2):242-251.

9. Tindall GT, Kovacs K, Horvath E, et al. Human prolactin-producing adenomas and bromocriptine: a histological, immuno-cytochemical, ultrastructural and morphometric study. J Clin Endocrinol Metab. 1982;55(6):1178-1183. doi: 10.1210/jcem-55-6-1178.

10. Kontogeorgos G, Sambaziotis D, Piaditis G, et al. Apoptosis in human pituitary adenomas: a morphological and in-situ end-labeling study. Mod Pathol. 1997;10(9):921-926.

11. Gen M, Uozumi T, Ohta M, et al. Necrotic changes in prolactinomas after long term administration of bromocriptine. J Clin Endocrinol Metab. 1983;59(3):463-479. doi: 10.1210/jcem-59-3-463.

12. Losa M, Franzin A, Mortini P, et al. Usefulness of markers of cell proliferation in the management of pituitary adenomas. Clin Sci (Lond). 1998;95(2):129-135.

13. Burian K, Pendl G, Salah S. The recurrence of pituitary adenoma after transfrontal, transphenoidal or 2-stage combined operation. Wien Med Wochenschr. 1970;120(47):833-836.

14. Кадашев Б.А., Трунин Ю.К., Корниенко В.И., и др. Поэтапное применение транскраниального и транссфеноидального хирургических доступов в лечении аденом гипофиза // Вопросы нейрохирургии им. Н.Н. Бурденко. - 1996. - №4. C. 6-10. [Kadashev BA, Trunin YuK, Kornienko VI, et al. Poetapnoye primeneniye transkranial'nogo i transsfenoidal'nogo khirurgicheskikh dostupov v lechenii adenom gipofiza. Burdenko's journal of neurosurgery. 1996;(4):6-10. (In Russ).]

15. Eguchi K, Kawamoto K, Uozumi T, et al. Effect of cabergoline, a dopamine agonist, on estrogen-induced rat pituitary tumors: in vitro culture studies. Endocr J. 1995;42(3):413-420. doi: 10.1507/endocrj.42.413.

16. Ben-Jonathan N, Hnasko R. Dopamine as a prolactin (PRL) inhibitor. Endocr Rev. 2001;22(6):724-763. doi: 10.1210/edrv.22.6.0451.

17. Radl DB, Zárate $S$, Jaita G, et al. Apoptosis of lactotrophs induced by D2 receptor activation is estrogen dependent. Neuroendocrinology. 2008;88(1):43-52. doi: 10.1159/000116117. 
18. Colao A, Lombardi G, Annunziato L. Cabergoline. Expert OpinPharmacother. 2000;1(3):555-574. doi: 10.1517/14656566.1.3.555.

19. Stefaneanu L, Kovacs K, Scheithauer BW, et al. Effect of dopamine agonists on lactotroph adenomas of the human pituitary. Endocr Pathol. 2000;11(4):341-352. doi: 10.1385/ep:11:4:341

20. Aoki MP, Aoki A, Maldonado CA. Sexual dimorphism of apoptosis in lactotrophs induced by bromocryptine. Histochem Cell Biol. 2001;116(3):215-222. doi: 10.1007/s004180100307.

21. Zornitzki T, Knobler H, Nass D, et al. Increased MIB-1/Ki67 labeling index as a predictor of an aggressive course in a case of prolactinoma. Horm Res. 2004;61(3):111-116. doi: 10.1159/000075375.

22. Matsuyama J. Ki-67 expression for predicting progression of postoperative residual pituitary adenomas: correlations with clinical variables. Neurol Med Chir (Tokyo). 2012;52(8):563-569. doi: $10.2176 / \mathrm{nmc} .52 .563$.

23. Ekramullah SM, Saitoh Y, Ohnishi T, et al. Effects of bromocriptine on staining indices of Ki-67 and proliferating cell nuclear antigen, and nucleolar organizer region number in pituitary adenomas. Neurol Med Chir (Tokyo). 1995;35(4):221-226. doi: 10.2176/nmc.35.221.

24. Lu C, Ren Z, Huan C, et al. The role of Ki-67 in women with a resistant prolactinoma: a retrospective analysis in 199 hospitalized patients over a period of 5 years. Pak J Pharm Sci. 2014;27(4 Suppl):1075-1081.

25. Delgrange E, Sassolas G, Perrin G, et al. Clinical and histological correlations in prolactinomas, with special reference to bromocriptine resistance. Acta Neurochir (Wien). 2005;147(7):751-757. doi: 10.1007/s00701-005-0498-2

26. Melmed S, Casanueva FF, Hoffman AR, et al. Diagnosis and treatment of hyperprolactinemia: an Endocrine Society clinical practice guideline. J Clin Endocrinol Metab. 2011;96(2):273-288. doi: 10.1210/jc.2010-1692.

27. Астафьева Л.И., Кадашев Б.А., Кутин М.А., Калинин П.Л. Осложнения лечения макропролактином агонистами дофамина // Вопросы нейрохирургии им. Н.Н. Бурденко. - 2011. T.75. - №2. - C. 41-50. [Astafeva LI, Kadashev BA, Kutin MA, Kalinin $\mathrm{PL}$. Complications of treatment of prolactinoma by dopamine agonists. Burdenko's journal of neurosurgery. 2011;75(2):41-50. (In Russ).]

28. Prevedello DM, Jagannathan J, Jane JA, et al. Relevance of high Ki-67 in pituitary adenomas. Case report and review of the literature. Neurosurg Focus. 2005;19(5):E1 1. doi: 10.3171/foc.2005.19.5.12.

29. Salehi F, Agur A, Scheithauer BW, et al. Ki-67 in pituitary neoplasms: a review. Part I. Neurosurgery. 2009;65(3):429-437. doi: 10.1227/01.NEU.0000349930.66434.82

30. Landolt AM, Keller PJ, Froesch ER, et al. Bromocriptine: Does it jeopardise the result of later surgery for prolactinomas? Lancet. 1982;2(8299):657-658. doi: 10.1016/s0140-6736(82)92756-8.

31. Faglia G, Moriondo P, Travaglini $P$, et al. Influence of previous bromocriptine therapy on surgery for microprolactinoma. Lancet. 1983;1(8316):133-134. doi: 10.1016/s0140-6736(83)91782-8.

32. DeLellis RA, Lloyd RV, Heitz PU, Eng C. Pathology and genetics of tumours of endocrine organs. 3rd ed. World Health Organization classification of tumours. Lyon: IARC Press; 2004

33. Zada G, Woodmansee WW, Ramkissoon S, et al. Atypical pituitary adenomas: incidence, clinical characteristics, and implications. J Neurosurg. 2011;114(2):336-344. doi: 10.3171/2010.8.JNS10290.

34. Chiloiro S, Doglietto F, Trapasso B, et al. Typical and atypical pituitary adenomas: a single-center analysis of outcome and prognosis. Neuroendocrinology 2015;101(2):143-150. doi: 10.1159/000375448.

35. Mete O, Lopes MB. Overview of the 2017 WHO Classification of Pituitary Tumors. Endocr Pathol. 2017;28(3):228-243. doi: 10.1007/s12022-017-9498-z.

36. Lopes MB. The 2017 World Health Organization classification of tumors of the pituitary gland: a summary. Acta Neuropathol. 2017;134(4):521-535. doi: 10.1007/s00401-017-1769-8.

Рукопись получена: 24.03.2020. Одобрена к публикации: 07.07.2020. Опубликована online: 10.08.2020.

\section{ИНФОРМАЦИЯ ОБ АВТОРАХ [AUTHORS INFO]}

*Астафьева Людмила Игоревна, д.м.н., ведущий научный сотрудник [Ludmila I. Astafyeva, MD, PhD]; адрес: ул. 4-я Тверская-Ямская, д. 16, Москва, Российская Федерация, 125047 [address: 16, 4th Tverskaya-Yamskaya street, Moscow 125047, Russia]; ORCID: http://orcid.org/0000-0003-4480-1902; eLibrary SPIN: 4209-4723; e-mail: Last@nsi.ru

Шишкина Людмила Валентиновна, к.м.н., ведущий научный сотрудник [Lyudmila V. Shishkina, MD, PhD], ORCID: http://orcid.org/0000-0001-7045-7223; SPIN-код: 6341-2050; e-mail: Lshishkina@nsi.ru

Кадашев Борис Александрович, д.м.н., профессор, ведущий научный сотрудник [Boris A. Kadashev, MD, PhD, Professor]; ORCID: http://orcid.org/ 0000-0001-8344-3381; eLibrary SPIN: 4157-6654; e-mail: kadashev@nsi.ru

Калинин Павел Львович, д.М.Н., ведущий научный сотрудник [Pavel L. Kalinin, MD, PhD];

ORCID: http://orcid.org/0000-0001-9333-9473; eLibrary SPIN: 1775-7421; e-mail: PKalinin@nsi.ru

Сиднева Юлия Геннадьевна, к.м.н., старший научный сотрудник [Julia G. Sidneva, MD, PhD]; ORCID: http://orcid.org/0000-0003-2733-5874; eLibrary SPIN: 5128-9059; e-mail: ysidneva@nsi.ru

Шарипов Олег Ильдарович, к.м.н., врач-нейрохирург [Oleg I. Sharipov, MD, PhD];

ORCID: http://orcid.org/0000-0003-3777-5662; eLibrary SPIN: 3279-0844; e-mail: osharipov@nsi.ru

Мельниченко Галина Афанасьевна, академик РАН, професcop, д.м.н. [Galina A. Melnichenko, MD, PhD, Professor]. ORCID: http://orcid.org/0000-0002-5634-7877; eLibrary SPIN: 8615-0038; e-mail: teofrast2000@mail.ru

\section{ЦИТИРОВАТЬ:}

Астафьева Л.И., Шишкина Л.В., Кадашев Б.А., Калинин П.Л., Сиднева Ю.Г., Шарипов О.И., Мельниченко Г.А. Изменение морфологической структуры гигантской пролактиномы на фоне лечения каберголином // Проблемы эндокринологии. — 2020. - T. 66. — №3. - C. 15-21. doi: https://doi.org/10.14341/probl12368

\section{TO CITE THIS ARTICLE:}

Astaf'eva LI, Shishkina LV, Kalinin PL, Kadashev BA, Sidneva JG, Sharipov OI, Melnichenko GA. Changes in the morphological structure of giant prolactinoma during treatment with cabergoline. Problems of Endocrinology. 2020;66(3):15-21. doi: https://doi.org/10.14341/probl12368 